\title{
A Mobile, Collaborative, Real Time Task List for Inpatient Environments
}

K.A. Michelson'; T. Ho'; A. Pelletier²; S. Al Ayubi²; F. Bourgeois'

${ }^{1}$ Boston Children's Hospital, Boston, MA, United States; ${ }^{2}$ Boston Children's Hospital, Innovation Acceleration Program, Boston, MA, United States

\section{Keywords}

Interdisciplinary communication, communication barriers, medical informatics, health applications

\section{Summary}

Background: Inpatient teams commonly track their tasks using paper checklists that are not shared between team members. Team members frequently communicate redundantly in order to prevent errors.

Methods: We created a mobile, collaborative, real-time task list application on the iOS platform. The application listed tasks for each patient, allowed users to check them off as completed, and transmitted that information to all other team members. In this report, we qualitatively describe our experience designing and piloting the application with an inpatient pediatric ward team at an academic pediatric hospital.

Results: We successfully created the tasklist application, however team members showed limited usage.

Conclusion: Physicians described that they preferred the immediacy and familiarity of paper, and did not experience an efficiency benefit when using the electronic tasklist.

\section{Correspondence to:}

Kenneth A. Michelson, MD

Division of Emergency Medicine, BCH 3066

Boston Children's Hospital

300 Longwood Ave.

Boston, MA 02115

Tel: (617) 355-6624

Fax: (617) 730-1024

Email: kenneth.michelson@childrens.harvard.edu
Appl Clin Inform 2015; 6: 677-683

http://dx.doi.org/10.4338/ACl-2015-05-CR-0050

received: May 11, 2015

accepted in revised form: September 20, 2015

published: November 18, 2015

Citation: Michelson KA, Ho T, Pelletier A, Al Ayubi S,

Bourgeois F. A mobile, collaborative, real-time task list for inpatient environments. Appl Clin Inform 2015; 6: 677-683

http://dx.doi.org/10.4338/ACl-2015-05-CR-0050 


\section{Introduction}

Communication errors are leading causes of errors in inpatient settings [1]. Caring for hospitalized patients with many team members in an inpatient environment is complex, and the reasons underlying communication errors are numerous [2]. Trainees are generally responsible for the day-to-day management of patients. Trainees often have many tasks, divide their attention between multiple activities and have high demands around communication and documentation [3,4]. The use of electronic tools may increase efficiency and facilitate communication in inpatient environments [5].

Physicians often use paper checklists to track their daily tasks and facilitate handoffs [6-8]. Paper is extremely convenient, but is static, redundant, unstandardized, unshared, and insecure $[9,10]$. Electronic medical records (EMR) alone may not add efficiency, improve communication, or reduce costs $[11,12]$. Commercial applications are available to emulate these paper task lists, but may not have security or privacy controls, or be supported by local institutions. Checklists have also been developed as part of workstation-based EMRs, but often lack mobile or live-tracking components [2, $6,13,14]$. Mobile tasklists have previously been successfully implemented [15].

Because of our dissatisfaction with the present system of task tracking and the lack of a privacycompliant shared checklist, we designed and implemented a secure, mobile application, called Tasklist, at Boston Children's Hospital (BCH), one of the nation's largest standalone pediatric hospitals. More than 140 pediatric residents care for patients throughout the hospital. Although a comprehensive EMR exists, there is limited support for the creation of task lists. Currently residents have a structured handoff support document that is generated through the EMR, but most residents print it once daily and update by hand through the day. We sought to design, implement, and deploy an iOS7-based application meeting current privacy and security standards and would allow physician teams to create, update, and complete tasks in a distributed and real-time way. Our aim was to report on our experience with this tool compared to the existing paper standard.

\section{Methods}

\subsection{Application Development}

Application development began with two authors (KAM and TH) sketching an electronic workflow that mirrored a familiar ward workflow, augmented by task sharing and logging capabilities.

We developed the Tasklist app on the iOS7 platform (Apple Corp., Mountain View, CA, United States). About $80 \%$ of mobile devices connected to the BCH network daily are iOS devices. Tasklist was linked to the hospital's clinical systems including PowerChart (Cerner Corp., Kansas City, MO, United States) and EPIC (Epic Corp., Verona, WI, United States) only through downloading. Inpatient teams downloaded Tasklist on their own mobile devices using the BCH Application Store; the application was only accessible to download while on the hospital network or virtual private network (VPN). We ensured that all team members had an iOS-based device by loaning devices to team members who did not have one.

The application's data were stored in a central database of tasks administered by the Tasklist team and residing on the Boston Children's Hospital computer network, where the tasks remained archived. Data retrieval, insertion, update, and deletion were performed using Java RESTful web service, while Cerner Millennium Objects (Cerner Corp., Kansas City, MO, United States) facilitated integration with Cerner EMR.

\subsection{Application Function}

After logging into Tasklist using a $\mathrm{BCH}$ enterprise account connected to the $\mathrm{BCH}$ lightweight directory access protocol authentication system, the home screen allowed the user to select their inpatient team. For the rest of the session, the user was associated with that team. Subsequently, a clinician was presented with the List View, a list of patients in that medical service and patient demographic data ( Figure 1A). This list was automatically populated from the hospital EMR. 
When a clinician selected a patient, Tasklist presented the Patient View, including patient demographics, a "sick flag" denoting whether the patient was deemed seriously ill, and the list of tasks associated with that patient ( $\triangleright$ Figure 1B). The sick flag was toggled on or off by clicking the icon. A new task was added by clicking the "+" button. Once a task was created, all clinical team members could immediately see the task and would have the ability to comment and update the state of that task. In the patient specific view, a user could change the task state to "In Progress" or "Complete." Once complete, tasks moved off of the Patient View to a completed tasks view. Tasks were removed after 12 hours in order to avoid cluttering the user's view with old, completed tasks.

After clicking a task in the patient view, Tasklist presented the Task View ( $\triangleright$ Figure 1C). From the Task View, a log of state changes to the task was viewable.

Clinicians could subscribe to notifications from Tasklist to get alerts when tasks were newly entered, updated or completed or when the patient sick flag was set. On task completion, the clinician completing the task had the option to send a notification to all team members if necessary. Clinicians could also request a summary of all open and completed tasks, which was e-mailed immediately to that clinician.

\subsection{Privacy and Security}

Compliance with Health Insurance Portability and Accountability Act standards and strict adherence to high-security policies was critical in designing the application, as Tasklist reported real patient data and associated protected health information.

The same hospital-wide username and password used for the EMR and email access were used to $\log$ in to the Tasklist application. In order to make Tasklist more usable, a full login of user name and password was required only once daily, after which a four-digit user-defined personal identification number (PIN) was used to access the application for the subsequent 24 hours. After that 24 hour period, the user would need to perform a full log in and set a new PIN.

Use of the application was restricted to devices without unauthorized operating system modifications ("jail breaking"), with a passcode enabled, and with an appropriate hospital security certificate. Additionally, Tasklist only functioned when the device was connected through $\mathrm{BCH}$ virtual private network or to the $\mathrm{BCH}$ wireless network. Tasklist had an automatic 5-minute-inactive logoff function to prevent the application from continuously displaying private data. Screenshots and cloud backup were disabled from within the application. No data were cached on the devices.

\subsection{Pilot Design}

A pilot period of use was conducted with a goal of obtaining verbal feedback on its use. We conducted a 10-day trial of Tasklist on a representative inpatient general pediatric ward. The ward was selected for its convenience, which cares for a median 18 (interquartile range 15-20) children at a time. The ward housed two teams, each with an attending physician in charge, a single supervising resident in their second or third year of pediatric residency, and two first-year pediatric residents. Of the eight total team members, two did not have an iOS device and received a loaner device. The team has physician and patient continuity from day-to-day. All team members obtained the Tasklist application prior to starting the pilot. The participants received a 20-minute tutorial on the use of Tasklist and were instructed to use the application in addition to the usual routine.

Our sole quantitative measurement was the frequency of task creation. We measured the number of tasks created and edited each day by user.

All other data were qualitative and unstructured. We assessed these qualitative reports of experiences from participants by eliciting in-person and e-mailed reports of what worked well and what did not work well, then summarizing them.

\section{Results}

Users created or edited a total of 234 tasks during the pilot. Tasklist events were created and edited with escalating frequency over the first several days of the pilot ( $>$ Figure 2 ). The team's supervising 
physician created or edited 209/234 (89\%) tasks. The application was unused over weekend days, followed by renewed use during weekdays. The maximum number of task items edited in a day was 87.

Team members reported that the use of Tasklist reinforced daily tasks for the team, it was easy to use, and took little time to become acquainted. Barriers to use included that paper was faster to use largely due to the time to acquire a wireless signal and log in and that team members already sat near one another so the application was less important as a communication tool than it may have been had the team been physically separated. Participants also desired Tasklist to be built into the EMR so they could seamlessly go between the computer-based EMR and mobile device-based Tasklist application to enter, edit, and complete tasks.

\section{Discussion}

We implemented a mobile, collaborative, real-time task list application and piloted it on a busy inpatient ward in a pediatric hospital. Based on the qualitative reports we elicited, clinicians found the application helpful in structuring discussion around patients. We overcame the security and privacy barriers of using clinicians' own devices. Additionally, the knowledge gained in building this mobile application was valuable experience for our information technology group in building future applications that require robust security measures.

Team culture of predominantly face-to-face communication and convenience of existing handwritten notes were major limitations in acceptance of a mobile collaborative Tasklist for maintaining and sharing tasks and notifying team members in place of paper. As part of physician training, trainees establish a personal, efficient system for tracking their own tasks, typically on paper. Paper is also a convenient method for most clinicians; the time needed to connect to a network and log in simply to add a task or check off a task as completed is by its nature longer than putting pen to paper. Entering tasks on an electronic device for this inpatient medical team was less efficient for clinicians than their current system of using paper and in person communication. Thus, although the team used Tasklist during the pilot, it did not reduce time spent on creating tasks. Use declined during the pilot, which was primarily due to the lack of a benefit to team performance. Different physicians staffed the unit over the weekend, and they did not receive the tutorial on the application, resulting in no use of the application for those days.

A future iteration of the mobile tasklist could serve clinicians by populating tasks in EMR documentation, provide other means of viewing tasks to the team, and would support other goals such as structured handoffs. Since team members use the EMR very frequently, integration between the EMR and a tasklist app could help address the inefficiencies of users populating two parallel systems with information. Additionally, a goal of a future iteration is reduction in time to task creation. This could be achieved through the option to create templates of tasks or bundles of tasks that often occur together. Additionally, network improvements could reduce latency within the application.

Limitations to this pilot work include the absence of survey or other systematically acquired quantitative data to support a change in practice. We could not measure how often specific features were used other than task creations and edits. Furthermore, we did not measure the acceptability or use patterns of paper checklists. However, the goal of this study was to report on our experience in designing this application, and not to demonstrate its superiority to the present system.

In summary, we overcame the security and privacy barriers to implementing a task checklist application on mobile devices. Usability enhancements, simplification of workflow, and integration with the EMR could augment acceptance of a mobile checklist application for a co-located medical team.

\section{Clinical Relevance Statement}

This work is relevant to the clinician as mobile checklist applications have the potential to enhance the clinician's ability to accomplish his or her tasks more efficiently and to facilitate teamwork by having a list shared with a team. This work was tested in a live clinical environment and thus has applicability to practicing clinicians. 


\section{Conflicts Statement}

The authors declare they have no conflicts of interest in the research.

\section{Protection of Human and Animal Subjects}

The study was performed in compliance with the World Medical Association Declaration of Helsinki on Ethical Principles for Medical Research Involving Human Subjects, and was exempted by the Boston Children's Hospital Institutional Review Board. 


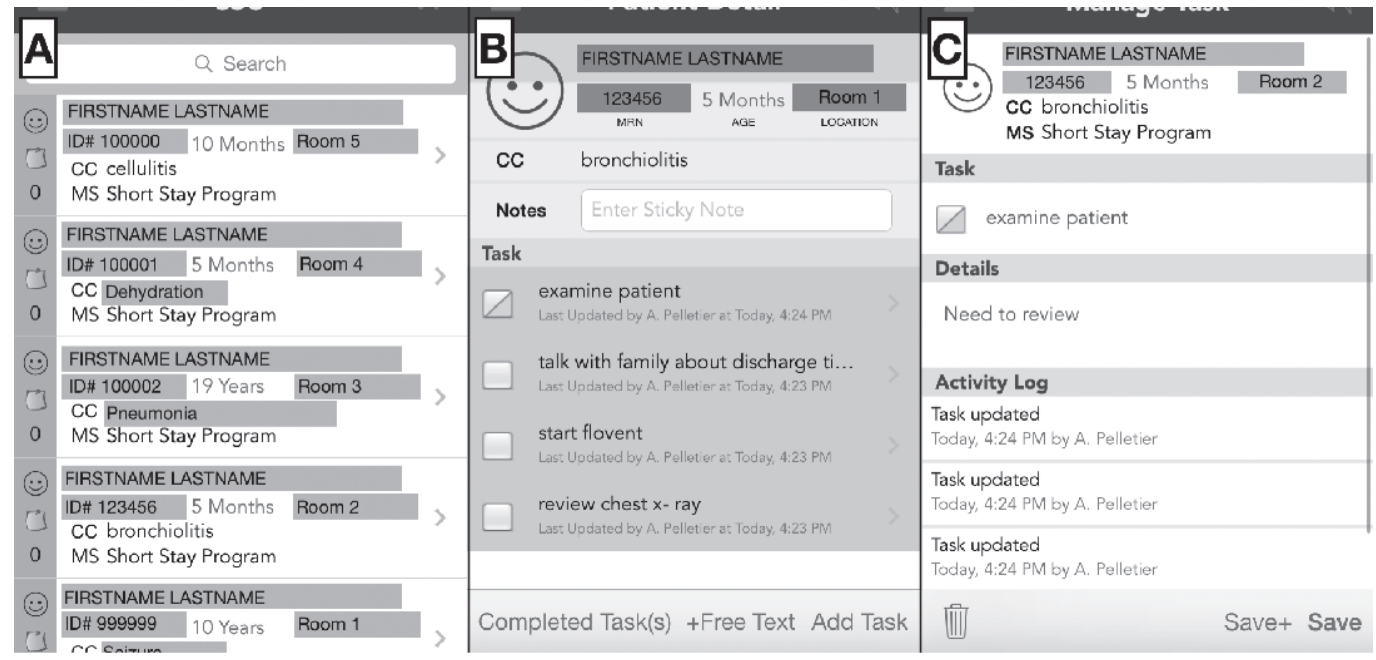

Fig. 1 Screenshots from Tasklist show the three main screens available to users. The List View view shows a list of all patients on a ward, including demographics, location, the patient's chief complaint, and medical service (panel A). Touching anywhere on a patient's panel opens the Patient View (panel B). Empty checkboxes denote pending tasks, while half-filled boxes indicate in-progress tasks. Clicking on the text of a task opens the Task View, which allows a user to add task details, view a log of changes, or delete the task (panel C). Clicking "Save+" updates a task and notifies all other users, while clicking "Save" updates a task without broadcasting an update.

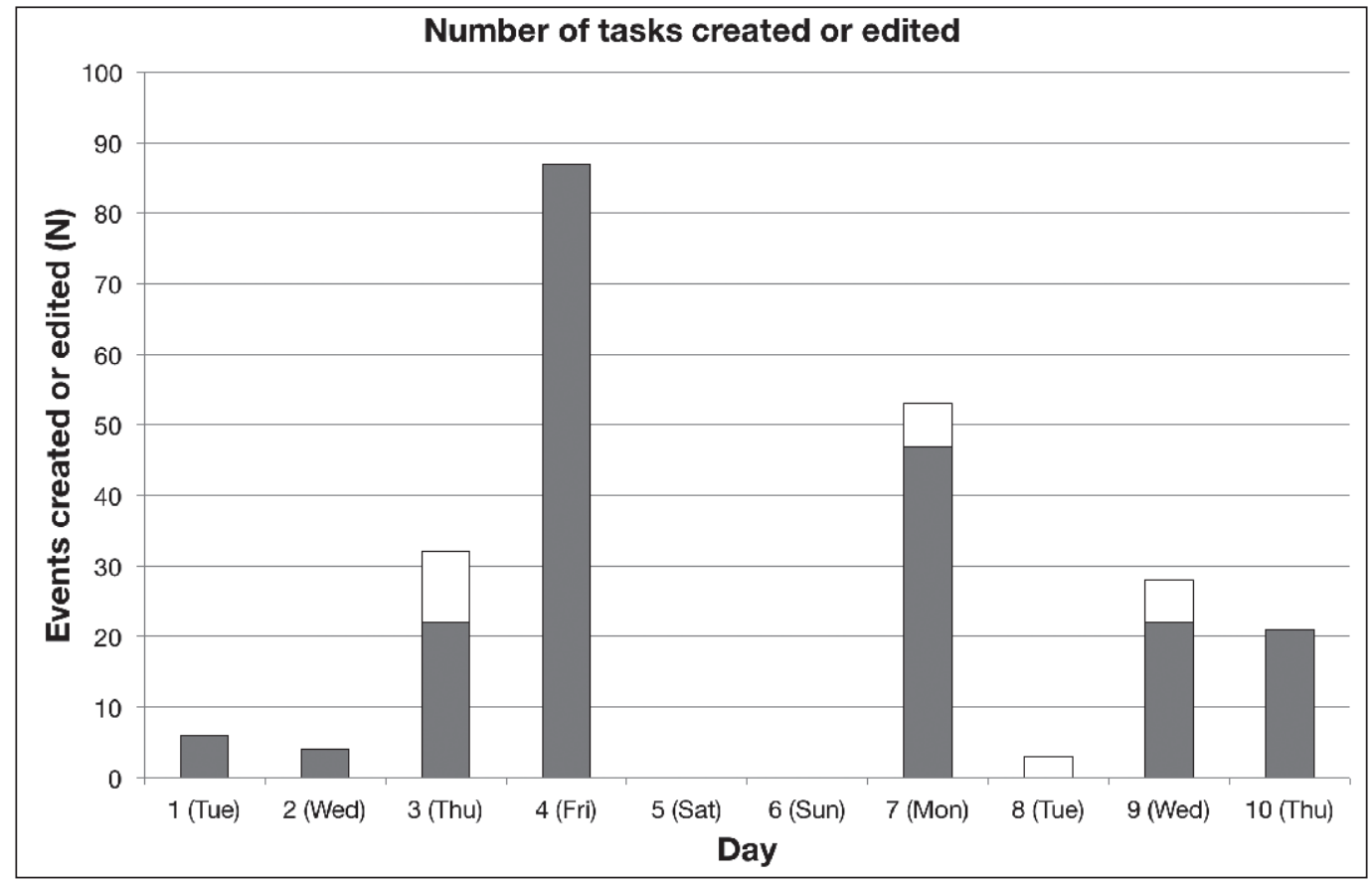

Fig. 2 The number of tasks created or edited during a 10-day pilot illustrates increasing adoption over several days by physicians trained to use Tasklist (gray: tasks created or edited by a supervising physician; white: tasks created or edited by a non-supervising physician). Editing included changing the status of a task between pending, in-progress, and complete. No tasks were created or edited during a weekend in which physicians untrained on Tasklist took care of patients. 


\section{References}

1. Joint Commission. Sentinel Event Data - Root Causes by Event Type. http://www.jointcommission.org/ sentinel_event_statistics/.

2. Starmer AJ, Sectish TC, Simon DW, Keohane C, McSweeney ME, Chung EY, Yoon CS, Lipsitz SR, Wassner AJ, Harper MB, Landrigan CP. Rates of medical errors and preventable adverse events among hospitalized children following implementation of a resident handoff bundle. JAMA 2013; 310(21): 2262-2270.

3. Tipping MD, Forth VE, Magill DB, Englert K, Williams MV. Systematic review of time studies evaluating physicians in the hospital setting. J Hosp Med 2010; 5(6): 353-359.

4. Morton JM, Baker CC, Farrell TM, Yohe ME, Kimple RJ, Herman DC, Udekwu P, Galanko JA, Behrns KE, Meyer AA. What do surgery residents do on their call nights? Am J Surg 2004; 188(3): 225-229.

5. Lobo MJ, Crandley EF, Rumph JS, Kirk SE, Dunlap NE, Rahimi AS, Turner AB Jr, Larner JM, Read PW. Pilot Study of iPad Incorporation Into Graduate Medical Education. J Grad Med Educ 2013; 5(1): 142-144.

6. Stein DM, Wrenn JO, Stetson PD, Bakken S. What "to-do" with physician task lists: clinical task model development and electronic health record design implications. AMIA Annu Symp Proc 2009; 2009: 624-628.

7. Van Eaton EG, Horvath KD, Lober WB, Rossini AJ, Pellegrini CA. A randomized, controlled trial evaluating the impact of a computerized rounding and sign-out system on continuity of care and resident work hours. J Am Coll Surg 2005; 200(4): 538-545.

8. Stein DM, Vawdrey DK, Stetson PD, Bakken S. An analysis of team checklists in physician signout notes. AMIA Annu Symp Proc 2010; 2010: 767-771.

9. Collins SA, Stein DM, Vawdrey DK, Stetson PD, Bakken S. Content overlap in nurse and physician handoff artifacts and the potential role of electronic health records: a systematic review. J Biomed Inform 2011; 44(4): 704-712.

10. Campion TR, Weinberg ST, Lorenzi NM, Waitman LR. Evaluation of Computerized Free Text Sign-Out Notes: Baseline Understanding and Recommendations. Appl Clin Inform 2010; 1(3): 304-317.

11.Victores AJ, Coggins K, Takashima M. Electronic health records and resident workflow: A time-motion study of otolaryngology residents. Laryngoscope 2015; 125(3): 594-598.

12. Himmelstein DU, Wright A, Woolhandler S. Hospital computing and the costs and quality of care: a national study. Am J Med 2010; 123(1): 40-46.

13. Rashba HW. TRAKIT--a computer-assisted personal to-do list. Pediatrics 1989; 83: 1007-1010.

14. Van Eaton EG, Horvath KD, Lober WB, Pellegrini CA. Organizing the transfer of patient care information: the development of a computerized resident sign-out system. Surgery 2004; 136(1): 5-13.

15. Pryss R, Mundbrod N, Langer D, Manfred R. Supporting medical ward rounds through mobile task and process management. Inf Syst E-bus Manag 2015; 13 (1): 107-146. 